\title{
High rate fast timing Glass Resistive Plate Chambers for the high $\eta$ CMS muon detectors
}

\author{
L. Mirabito*† \\ Institut de Physique Nucleaire de Lyon-UCBL-IN2P3, Lyon,France \\ E-mail:laurent.mirabito@cern.ch
}

\begin{abstract}
With the increase of the LHC luminosity foreseen in the high luminosity version, some detectors currently used in CMS will not sustain high fluences and some need to be replaced. The new ones could provide time information and could reduce the data ambiguity due to the expected high pileup. RPC using low-resistivity materials are proposed to equip the very forward region of the CMS detector. In their single-gap version we will show that they can stand rates of few $\mathrm{kHz} / \mathrm{cm}^{2}$. We also demonstrate that using multi-gap glass RPC, a time resolution of about $60 \mathrm{ps}$ is achieved.
\end{abstract}

38th International Conference on High Energy Physics

3-10 August 2016

Chicago, USA

* Speaker.

$\dagger$ on behalf of CMS muon group 


\section{Introduction}

During the Phase II of the LHC program (HL-LHC) the instantaneous luminosity in CMS experience is expected to increase by a factor 10 or more. In this context, a new generation of low-resistivity glass RPC (LR-GRPC) able to sustain large irradiation rates has been developed at IPNL and Tsinghua University [6]. These detectors are proposed to equip the high $\eta$ muon stations (Fig 1) $(1.8<|\eta|<2.4)$. They should be able to withstand a particle fluence that can reach up to 2 $\mathrm{kHz} \cdot \mathrm{cm}^{-2}[1]$

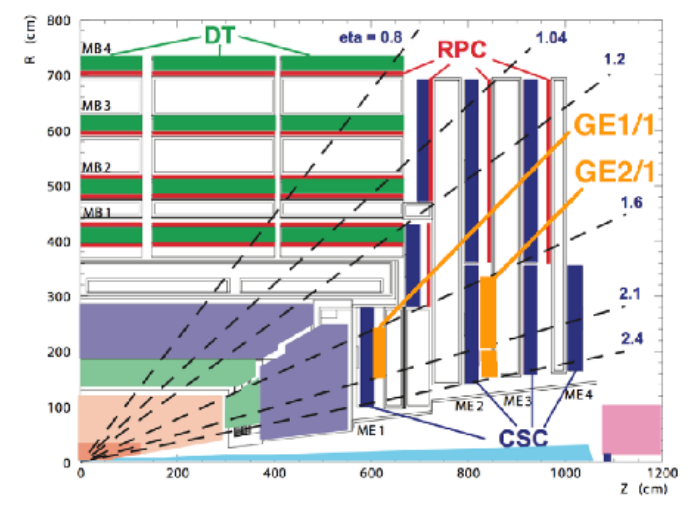

Figure 1: Layout of one quadrant of CMS. The slots RE3/1 and RE3/2 are expected to be instrumented by RPC chambers for HL-LHC upgrade.

The technology is under validation since 2014 using muon beams at CERN PS and SPS lines, eat HZDR and in the Gamma Irradiation Facilities (GIF/GIF++). We present here new results from these tests and describe the different prototypes proposed for the CMS upgrade program.

\section{Validation of the rate capabilities of low resistivity glass RPC (LR-GRPC)}

The single gap LR-GRPC detectors use low-resistivity glass plates as electrodes. The new glass, developed by Tsinghua University[3], has a resistivity in the range $10^{9}-10^{10} \Omega . \mathrm{cm}$ and a very high surface uniformity, with a roughness below $10 \mathrm{~nm}$. Largest plates are $30 \times 30 \mathrm{~cm}^{2}$ with a thickness in the 0.5-2 mm range. Chambers are made of two, $1 \mathrm{~mm}$ thick, low resistivity, plates covered with a colloidal graphite coating (surface resistivity of a few $\mathrm{M} \Omega / \square$ ) separated by a gas gap of $1.2 \mathrm{~mm}$. Spacers made of fiber glass and ceramic are used to maintain uniform the distance between the two layers. To operate the detectors a gas mixture made of TFE(93\%), CO2(5\%) and SF6(2\%) is normally used, but the standard mixture used in CMS RPC muon system (95.2/4.5/0.3) is also used in high irradiation tests. The detectors are read using the CALICE SDHCAL [5] readout system based on the HARDROC2 Asic [2] equipping a board of 1536 pads of $1 \times 1 \mathrm{~cm}^{2}$.

A telescope made of 4 LR-GRPC and one GRPC with high-resistivity glass was exposed into two CERN SPS muon beam: the $\mathrm{H} 2$ Line with muons of $150 \mathrm{GeV}$ and an intensity in $10^{3}$ $10^{7}$ muons/spill (4.5 s), the $\mathrm{H} 4$ line in the Gamma Irradiation facility where it is exposed to an additional $10 \mathrm{TBq}{ }^{137} \mathrm{Cs}$ source. Figure 2 exhibits a high voltage working point of $\sim 7 \mathrm{kV}$ with 95 $\%$ efficiency for LR-GRPC in a low intensity beam of the H4 line. 


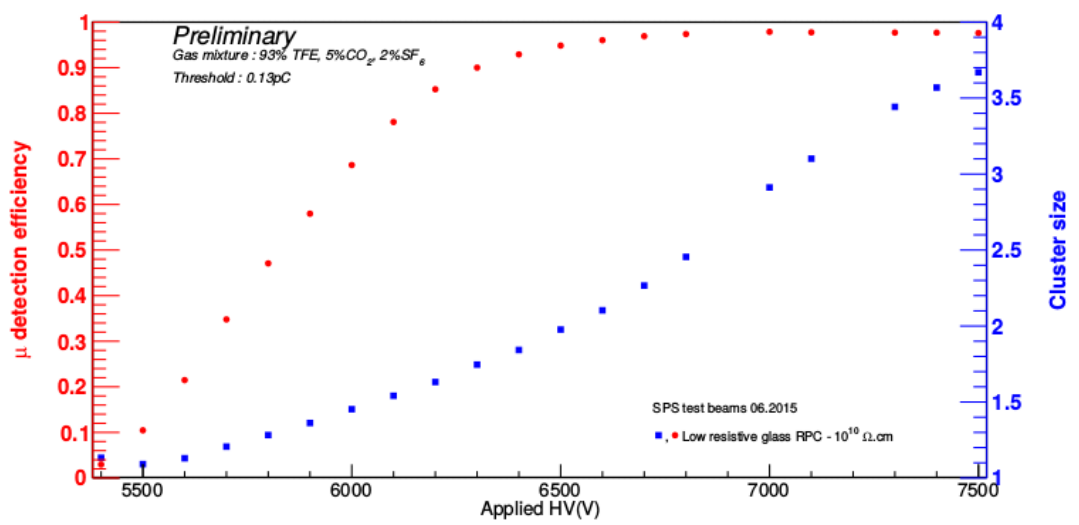

Figure 2: Characteristics (Efficiency and pad multiplicity vs HV) of LR-GRPC measured at SPS at low rate.

On the $\mathrm{H} 2$ line, the muon beam is concentrated in a $11 \times 11 \mathrm{~cm}^{2}$ Gaussian shape. The rate is averaged on the whole surface and a scan in intensity is performed (Fig. 3). The low resistivity chambers sustain a fluence of $7 \mathrm{kHz} / \mathrm{cm}^{2}$ with an efficiency above $70 \%$ while the standard glass detector efficiency declines above $0.2 \mathrm{kHz} / \mathrm{cm}^{2}$.

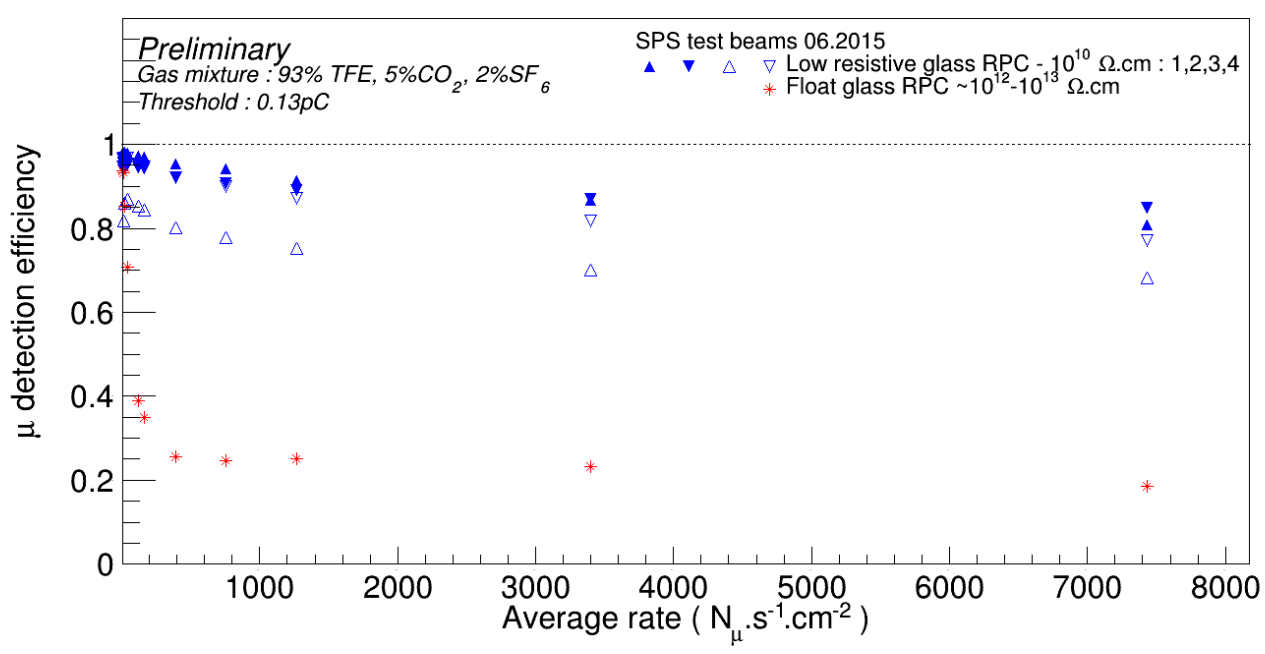

Figure 3: Efficiency vs the muon rate in intensive beam at SPS

On the $\mathrm{H} 4$ line at $\mathrm{GIF++}$, an intensive gamma irradiation is uniformly distributed and a low intensity muon beam is used to measure the efficiency. The gamma conversion factor is estimated by GEANT4 simulation to be 3.1 clusters/1000 photons. The CMS RPC gas mixture in this measurement contains 7 times less SF6 (electron quencher) than in SPS.The LR-GRPC efficiency (Fig. 4) remains above $90 \%$ for rate below $1 \mathrm{kHz} \cdot \mathrm{cm}^{-2}$. It drops below $70 \%$ for fluence above $2 \mathrm{kHz} \cdot \mathrm{cm}^{-2}$. In the case of a standard glass RPC, it drops for a fluence above $600 \mathrm{~Hz} \cdot \mathrm{cm}^{-2}$.

In this second test, the single particle charge is higher due to a lower quencher proportion and the global space charge is also higher since the whole chamber is irradiated. This explains the differences between the rate capabilities. The achieved performances $\left(\varepsilon>70 \%\right.$ at $\left.2 \mathrm{kHz} . \mathrm{cm}^{2}\right)$ are 


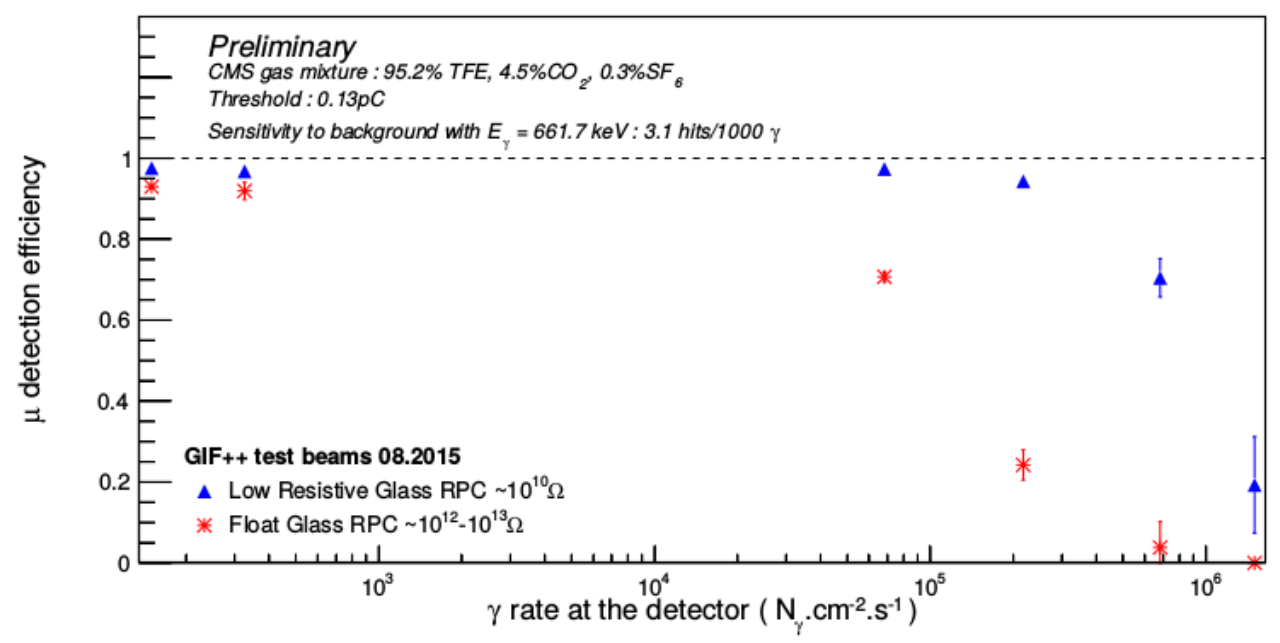

Figure 4: Efficiency vs the gamma rate in GIF++

nevertheless enough for the CMS forward chambers upgrade since planes made of 2 GRPC gaps readout simultaneously are considered.

One limitation of this technology is that large sizes of low resistivity glass plates cannot be built due to manufacturing constraints. Two methods were proposed to assemble smaller glass plates into large LR-GRPC chamber, gluing or mechanical fixation. Figure 5 shows two large prototypes built with those two techniques. Two GRPC made of glass using the second technique are stacked in a cassette with a strip-based print circuit board in between. Both prototypes were tested with cosmic muons and they both reach an efficiency plateau above $95 \%$ at $6.9 \mathrm{kV}$, as expected from the small prototypes studies.
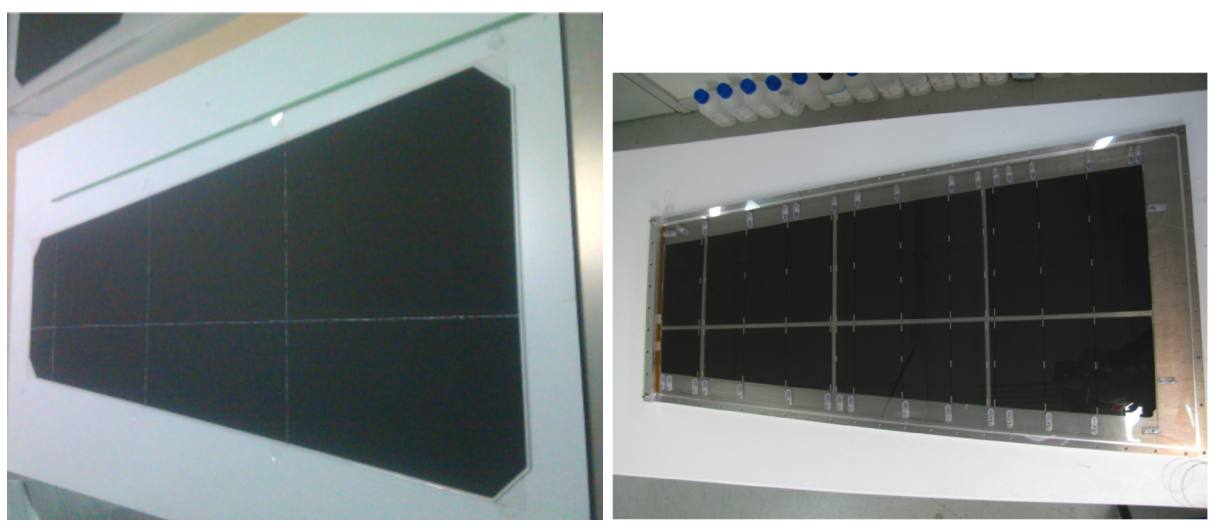

Figure 5: Glued (left) and assembly (right) prototype of low resistivity GRPC for RE3 CMS muon stations

\section{Fast timing}

\subsection{Electronic development}

The RPC in general, and more particularly the multi-gap ones, are excellent fast timing detectors. 
To exploit this feature a dedicated electronic readout system needs to be developed. We propose a special PCB (see Fig 6 left) that hosts $4.0 \mathrm{~mm}$ pitch strips read out by a 32-channel PETIROC ASIC [4], known for its low jitter ( $<20$ ps for Q $>150 \mathrm{fC})$.

Each end of a strip is connected to a channel of a unique ASIC. A 24-channel TDC, with a time resolution of about $25 \mathrm{ps}$, is used to measure the arrival time of the signal issued from PETIROC. This architecture allows to reconstruct the signal position along the strips from the difference of time arrival on both ends. The geometrical precision is depending only on the electronic resolution. The time resolution of the PCB is tested by injecting a few thousands of times, charges in several test points located on the strips. The result of this test on one strip is shown in Fig. 6 (right). The time distribution is fitted by a gaussian function. Repeated on all strips the achieved resolution is below 35 ps for an injected charge of $5 \mathrm{pC}$. Several tests with lower injection and with detectors are on going.
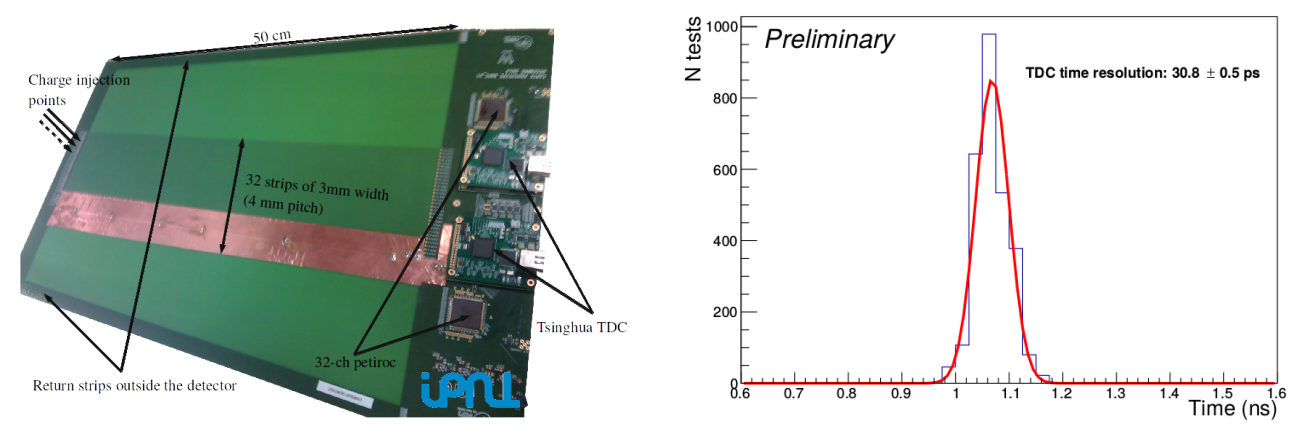

Figure 6: Left:Strip-based timing PCB, the PCB is slided between 2 RPC detector and both detector signals are read on the same strips. Right: example of time measurement with a charge injection of $5 \mathrm{pC}$ on one strip

\subsection{Multi gap LR-GRPC performances}

Several multi gap LR-GRPC chambers have already been built and operated in high rate beams [3]. More recently, a larger size multi gap GRPC with 5 gaps of $250 \mu \mathrm{m}$ was built with glued glasses (Fig. 7).

The detector is operated with a gas mixture of $85 \%$ TFE, $5 \%$ Isobutane and $10 \%$ SF6. Both charge and time are registered. The efficiency reaches a plateau above $95 \%$ at $6.8 \mathrm{kV}$ (Fig. 8), the glued area causes an efficiency drop of less than $2 \%$. The noise is kept below $1 \mathrm{~Hz} . \mathrm{cm}^{-2}$ and the time resolution is stable at about $60 \mathrm{ps}$.

It was exposed to high intensity e beam at HZDR. Figure 9 shows the rate capability of the MRPC. The efficiency and the time resolution are stable up to $30 \mathrm{kHz}$.

\section{Conclusion}

The properties of low resistivity GRPC were validated during multiple test beam campaigns. They remain efficient ( $>90 \%$ ) when exposed to a gamma rate of a few $\mathrm{kHz}$. In all condition those thingap chambers can be operated with a high voltage of the order of $7 \mathrm{kV}$. This is an interesting 

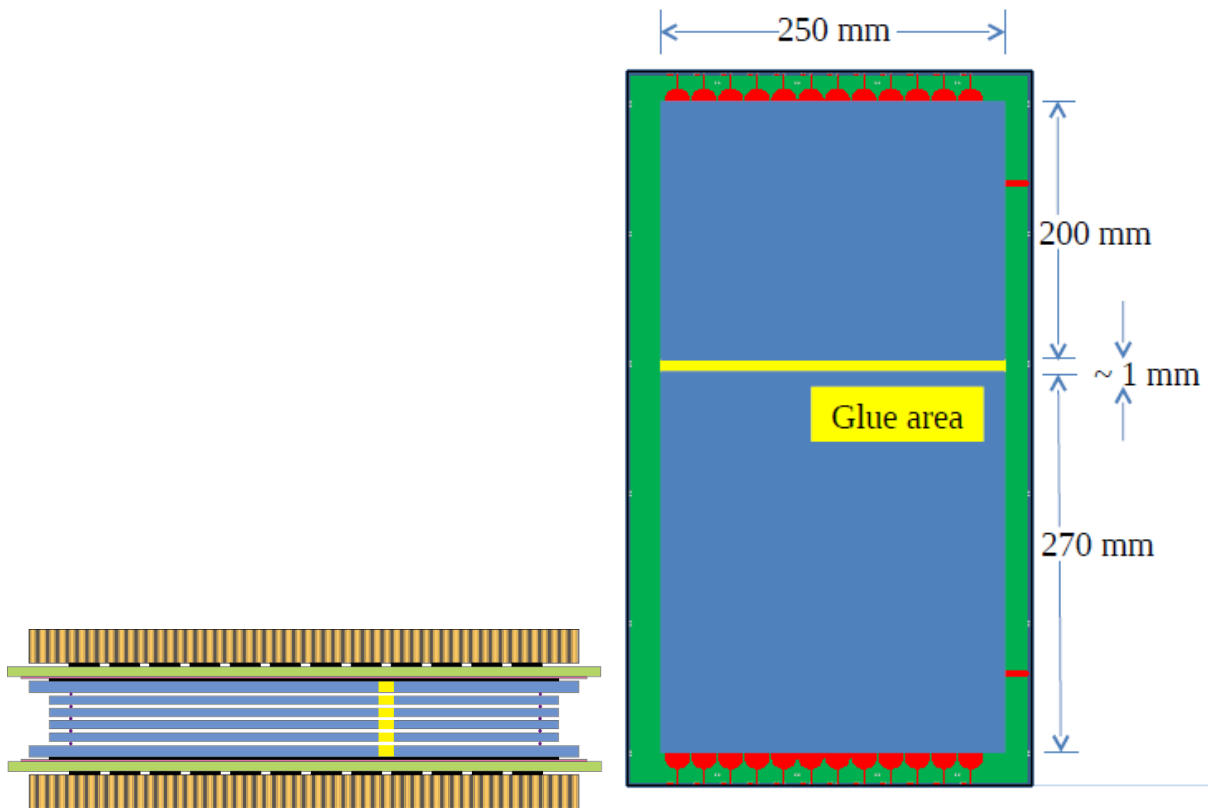

Figure 7: The multi-gap RPC design. Two glass are glued and 6 planes are stacked, separated by fishing lines

property for the mitigation of background at HL-LHC. Different alternative designs are proposed for the upgrade of CMS muon detector in the forward regions. Prototypes are built to validate the mechanical design, as well as the electronic readout.Very promising multi-gap detectors reached a time resolution of $60 \mathrm{ps}$. A more systematic validation campaign of large size prototypes is ongoing in 2016. At the same time an ageing test is ongoing in CERN Gamma Irradiation Facility since October 2015, with regular beam test campaigns to validate long term stability of this technology.

\section{References}

[1] CMS Coll. Technical Proposal for the Phase-II Upgrade of the CMS Detector. CERN-LHCC-2015-010/LHCC-P-008/CMS-TDR-15-02. 2015

[2] F.Dulucq et al. HARDROC: Readout Chip for CALICE/EUDET Digital Hadronic Calorimeter. NSS/MIC,2010IEEE,pp.1678-1683. 2010.

[3] J. B. Wang et al. Development of Multi-Gap Resistive Plate Chambers with Low-Resistive Silicate Glass Electrodes for Operation at High Particle Fluxes and Large Transported Charges. Nucl. Instrum. Meth. A 621. 2010.

[4] J.Fleury et al. Petiroc, a New Front-End ASIC for Time of Flight Application. IEEE Nuclear Science Symposium and Medical Imaging Conference NSS/MIC. 2013.

[5] M. Bedjidian et al. Performance of Glass Resistive Plate Chambers for a High Granularity Semi-Digital Calorimeter. JINST6 P02001. 2011.

[6] Y. Haddad et al. High Rate Resistive Plate Chamber for LHC Detector Upgrades. Nucl. Instr. Meth. Phys. Res. A718 p 424-426. 2013. 


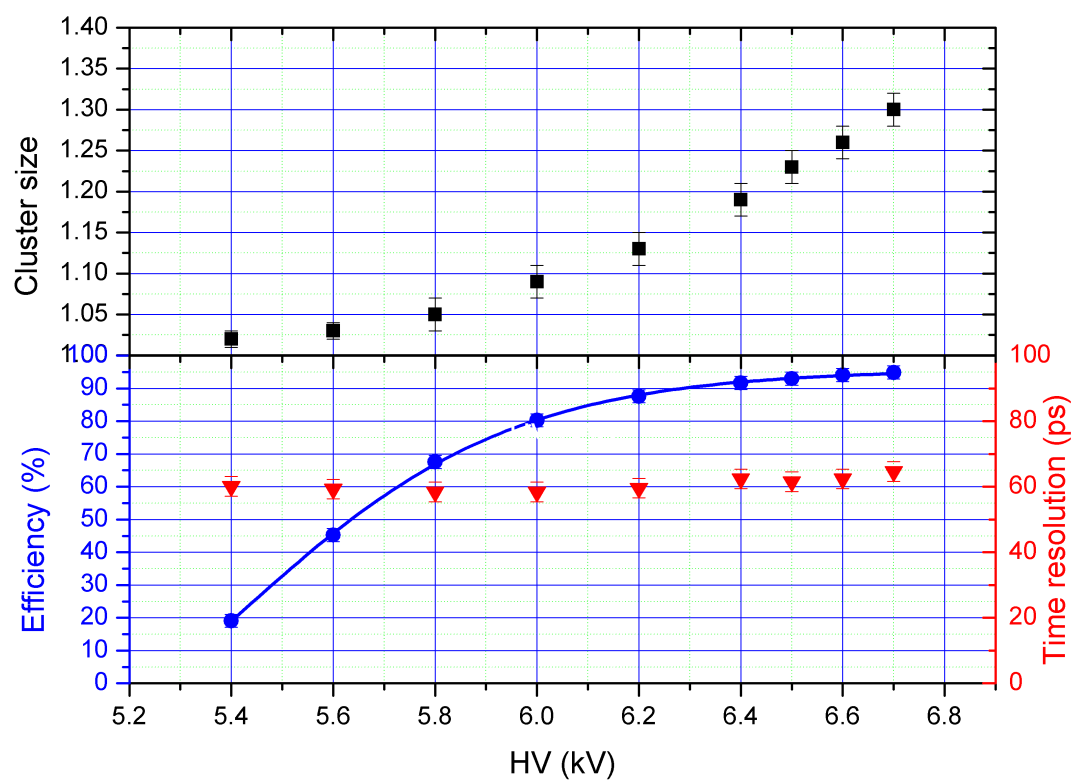

Figure 8: Performances of the MRPC

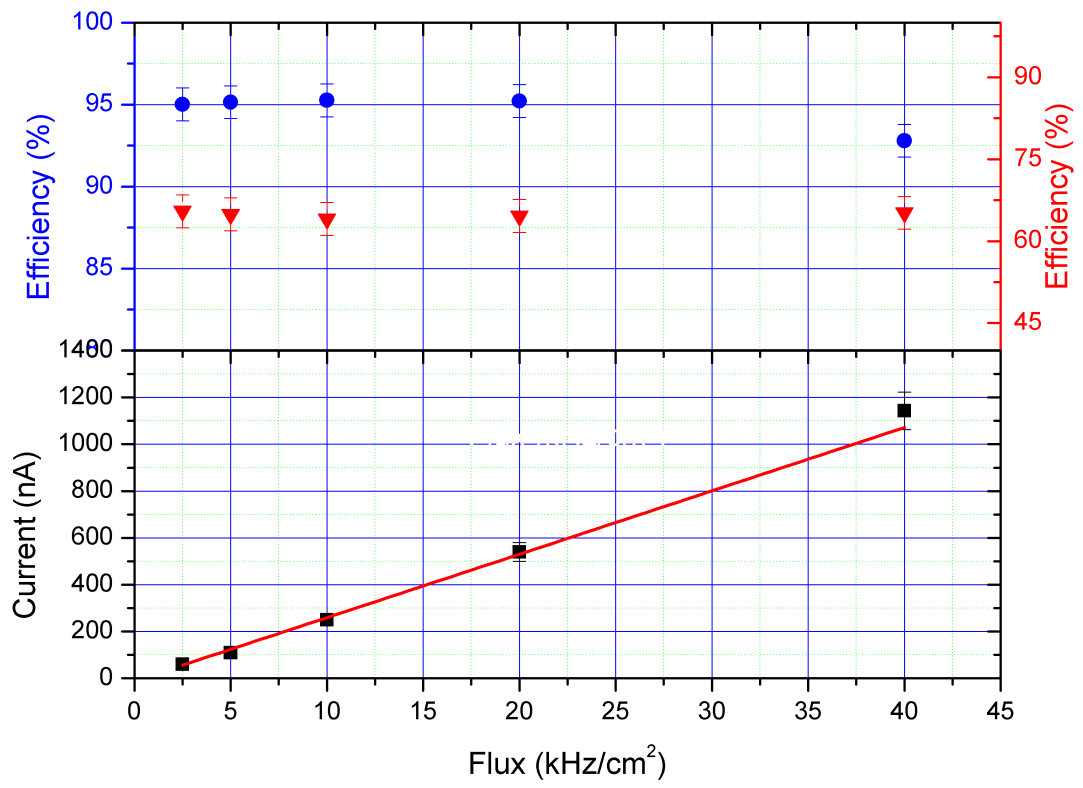

Figure 9: Rate capabilities of the MRPC: the current measured is proportional to the rate,i.e no saturation is observed 